\title{
Witness Gun for the Argonne Wakefield Accelerator
}

\author{
J. POWER, J. SIMPSON and E. CHOJNACKI
}

\author{
Argonne National Laboratory, Argonne, IL, 60439, USA
}

\section{Abstract}

In order to probe the wakefields of the Argonne Wakefield Accelerator (AWA) a beam of medium energy $(4-6 \mathrm{MeV})$ and relatively low charge $(100 \mathrm{pC}-1 \mathrm{nC})$ is needed. Two options for a witness gun meeting these specifications are currently under study. The first is a conventional iris loaded multicell gun. The second is a dielectric-lined cylindrical cavity operating in a pure $\mathrm{TM}_{01 \mathrm{n}}$ mode. Since a more accurate probing of the wakefields can be obtained by improving the witness beam quality, this factor of merit will determine which gun is better suited for the experiment. It is thought that a brighter beam may be obtained using the dielectric gun. Since the dielectric gun will operate in a single accelerating mode, higher order modes will not be present. This in turn may lead to a slower emittance growth. This potential benefit comes at the cost of a lower cavity $Q$ and dielectric charging considerations. Details of design, bench test and simulation results will be presented.

\section{INTRODUCTION}

The AWA group is considering two options for an electron gun for generation of a witness beam for use in the AWA project. After briefly describing why we are considering both options, the physical layout of each accelerating structure will be presented. Simulation results done with PARMELA will then be presented here for both options in an attempt to compare the guns. Bench test have already been done on the dielectric-lined gun using a network analyzer and a relatively high cavity $Q$ was found. The paper will end with some concluding comments about the future course for the AWA witness gun.

\section{MOTIVATION}

It is shown ${ }^{(1)}$ that two major causes of emittance growth are due to space charge and the nonlinearities present in the fields of an accelerating structure. For this reason, we feel there may be some benefit in using a structure operating in a pure $\mathrm{TM}_{01 \mathrm{n}}$ mode. The fields in the dielectric-lined cavity have be calculated ${ }^{(2)}$ and are very nearly linear in the region of acceleration. Whereas conventional slow wave structures using irises create large nonlinearities in the region of the irises. However, since the AWA project MUST have a functioning witness gun for its experiments, we have decided to also design a conventional copper cavity, for insurance purposes. The deign being studied is a scaled up version of the MARK IV accelerator.

\section{GUN CONSTRUCTION}

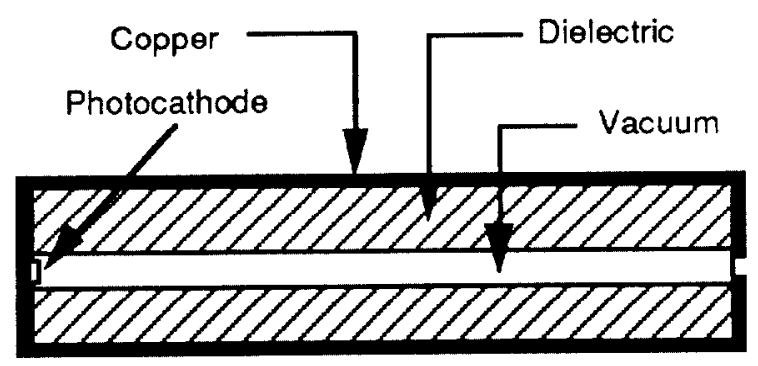

(a)

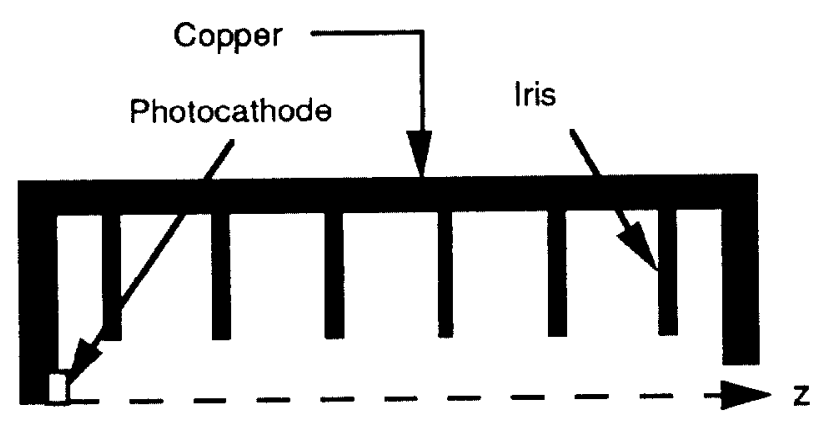

(b)

Fig. 1) Schematic views of both guns. a) Dielectric-lined gun b) 6 cell, Mark IV gun

\section{A. Dielectric Gun}

For our initial test cases we designed the dielectric gun to run in a $\mathrm{TM}_{013}$ mode and we chose the dielectric to be quartz. Since the rf power available has a frequency of $1.3 \mathrm{GHz}$ and we will operate at a phase velocity of $98 \%$ the speed of light, all dimensions of the cavity are fixed. The inner radius of this gun is $1 \mathrm{~cm}$. The inner radius is the radius of the region labeled vacuum in Fig 1a. Solving for the outer radius by requiring the particular phase velocity we obtain a radius of $5.44 \mathrm{~cm}$. The outer radius is from the center of the vacuum section to the copper/dielectric boundary. Lastly, the length of the cavity is determined to be $32.57 \mathrm{~cm}$.

\section{B. Mark IV Gun}

The Mark IV accelerator (an s-band structure) was the predecessor to the two mile accelerator at SLAC. We chose this design for several reasons. Since we will be running off 
the same modulator used to drive the preaccelerator ${ }^{(3)}$,we will have limited power available to operate the witness gun, about 4-6 MW. Due to both power and spatial limitations (not much room for the gun, about $1 \mathrm{~m}$ ) the gun will be operated in a standing wave mode. We also wanted an iris loaded cavity optimized for low bunch charge that would provide a $4-6 \mathrm{MeV}$ beam. With only minor modifications the Mark IV gun proved to be an excellent choice. Since the modulator will operate at $1.3 \mathrm{GHz}$ we needed to scale the dimensions of the original Mark IV gun up from s-band to l-band.The dimensions are as follows, the length is $46 \mathrm{~cm}$, the radius to the irises is 2.48 $\mathrm{cm}$, the radius to the outer walls is $9.05 \mathrm{~cm}$, the gap between irises is $7.68 \mathrm{~cm}$ and the thicknesses of the irises is $1.28 \mathrm{~cm}$.

\section{COMPARISION OF GUNS}

In 1992 we preformed a bench test to measure the cavity Q of the dielectric lined gun. Using alumina as the dielectric we made the measurements at room temperature with a network analyzer. The alumina tube was purchased from Coors and was quoted to have a loss tangent of $10^{-4}$. Given this value and the dimensions of the cavity, we expected a $Q$ of about 7000 . The result of the bench test however was a $Q$ of 12,500 . We felt that this was due to the particular sample we received from Coors having a loss tangent of $0.5 * 10^{-4}$. In any case, we now feel reasonably confident about being able to find high $Q$ dielectrics for our gun. For simulation purposes, however, we used a very conservative (low) value of $Q$.

Simulations are being carried out with a modified version of PARMELA ${ }^{(4)}$ for both guns. For our initial studies we have chosen quartz as our dielectric and to run in a $\mathrm{TM}_{013}$ mode $(\varepsilon=3.87 \& Q=6880)$. With this low value of $Q$ the dielectric gun is power consumptive, needing about $15 \mathrm{MW}$ to produce a $5.2 \mathrm{MeV}$ bunch energy. However, the other figures of merit, emittance, energy spread and bunch length show the beam to be of high quality (see table 1).

For the Mark IV gun we have chosen to use a 6 cell, standing wave gun operating in a $2 \pi / 3$ mode (see fig $1 b$ ). This gun gives acceptable beam quality at low power. For $4 \mathrm{MW}$ of power the emerging beam has an energy of $5.4 \mathrm{MeV}$ and sufficient beam quality (see table 1).

For comparison purposes a list of output parameters is given in table 1. A bunch charge of $100 \mathrm{pC}$ is being run through each gun. Both beams have an initial phase launch angle of $70^{\circ}$ and the size of the laser pulse on the photocathode is $2 \mathrm{~mm}$ in the dielectric gun and $1 \mathrm{~mm}$ in the Mark IV gun.

\begin{tabular}{|l|l|l|}
\hline & Dielectric & Mark IV \\
\hline & & \\
\hline Energy & $5.20 \mathrm{MeV}$ & $5.42 \mathrm{MeV}$ \\
\hline Emittance & $1.01 \mathrm{~mm}^{*} \mathrm{mrad}$ & $1.86 \mathrm{~mm}^{*} \mathrm{mrad}$ \\
\hline Energy spread & $0.69 \%$ & $3.28 \%$ \\
\hline Bunch length & $1.8 \mathrm{ps}$ & $3.57 \mathrm{ps}$ \\
\hline
\end{tabular}

Table 1) Comparison of the dielectric and Mark IV guns done with PARMELA.
Although the modified PARMELA code fully calculates space charge effects, it does not take wakefields into account. The primary effect of the wakefields are to increase the energy spread of the bunch. We have estimated the energy spread due to wakefields in both guns analytically. The energy spread calculated by PARMELA and due to the wakefields are given in table 2.

\begin{tabular}{|l|l|l|}
\hline & Dielectric & Mark IV \\
\hline & & \\
\hline Wakefields & $1.1 \%$ & $0.2 \%$ \\
\hline PARMELA & $0.69 \%$ & $3.28 \%$ \\
\hline Total & $1.79 \%$ & $3.48 \%$ \\
\hline
\end{tabular}

Table 2) Energy spread due to wakefields and PARMELA.

\section{THE FUTURE}

For the purposes of the AWA experiment we will probably have to use the Mark IV gun since the dielectric gun is too power consumptive. This does not rule it out altogether though. It still may be useful to someone looking at generation of a high quality beam of medium energy in a small space. As long as power is not your main limitation, the dielectric gun may prove to be a viable option. Since there are so many parameters that can be adjusted for the dielectric gun, We fully expect the beam parameters to improve further. Simulations on the dielectric gun will continue. The immediate plan is to first build the Mark IV gun so we have a working witness gun for the AWA project. We will then build and run the dielectric gun both at low power (4-6 MW) and at high power (15 MW) for further investigation into the feasibility of a dielectric lined electron gun.

\section{REFERENCES}

1.Kwang-Je Kim, Nuc. Inst.and Meth. in Phys. Research (1989) pg. 201-218, 'rf and space charge effects in laser-driven rf electron guns'

2. J. Power, Argonne HEP Division, Wakefield Note (1993)

3. M. Rosing et. al. , Argonne Wakefield Accelerator Update '92, Proc. 1992 Linear Accelerator Conf. (Ottawa,Ontario, Canada) Vol. 1, pg. 79

4. C. H. Ho, Ph.d.Thesis, UCLA (1992) 